\title{
Risk Factors for Hospital Readmission and Follow Up after NICU Dis- charge of Infants Born at Extremely Low Gestational Age in Metro- politan Melbourne
}

\author{
Tejas N Doctor ${ }^{1 *}$, Efrant Harnaen ${ }^{1}$, Bob Seith ${ }^{1,2}$, Kenneth Tan $^{3,4,5}$ and Simon Craig ${ }^{1,2,6}$ \\ ${ }^{1}$ Paediatric Emergency Department, Monash Medical Centre, Melbourne, Australia \\ ${ }^{2}$ Department of Medicine, Monash University, Melbourne, Australia \\ ${ }^{3}$ Monash Newborn, Monash Children's Hospital, Melbourne, Australia \\ ${ }^{4}$ Department of Pediatrics, Monash University, Melbourne, Australia \\ ${ }^{5}$ Hudson Institute of Medical Research, Melbourne, Australia \\ ${ }^{6}$ Murdoch Children's Research Institute, Melbourne, Australia
}

*Corresponding author: Dr. Tejas N Doctor, Advanced Trainee in Neonatal \& Perinatal Medicine and General Pediatrics; Neonatal Fellow Mercy Hospital for Women Heidelberg; Paediatric Emergency Department, Monash Medical Centre, Melbourne, Victoria, Australia, E-mail: drtejudoctor@yahoo.co.in

\begin{abstract}
Background: Extremely preterm infants, < 28 week of gestational age, (ELGANs) continue to need high levels of health-care post-discharge from the NICU. We studied postNICU hospital contacts of this highly vulnerable population and present data from infants treated in our health network.

Method: Retrospective cohort study of ELGANs treated at the tertiary NICU of Monash Health (2010-2013) and discharged within our network's catchment area. Demographics, inpatient, and outpatient information (initial NICU and subsequent admission up to 18 months of corrected age) were collated. Comparative analyses were performed for infants who were readmitted compared to those who were not.

Results: 41 of 76 (54\%) infants required hospitalization with 92 admission episodes. 81 episodes were general pediatric ward admissions while 11 were to the PICU/HDU. Viral bronchiolitis was the leading cause for admissions. Meningitis in neonatal period was more common among patients who required hospital re-admission.
\end{abstract}

Conclusions: Ex-ELGAN required readmission primarily due to viral bronchiolitis. In our study population, neonatal meningitis was more common in patients who required post NICU discharge hospital re-admission.

\section{Keywords}

Extremely Low Gestational Age Neonate (ELGAN), Hospital readmission, Long term follow up

\begin{abstract}
Abbreviations
AlHW: Australian Institute of Health and Welfare; ANZNN: Australia New Zealand Neonatal Network; APH: Ante Partum Hemorrhage; BPD: Broncho Pulmonary Dysplasia; CGH: Casey General Hospital; CPAP: Continuous Positive Airway Pressure; DGH: Dandenong General Hospital; ED: Emergency Department; ELGANs: Extremely Low for Gestational Age Newborns; GA: General Anaesthesia; IVH: Intraventricular Hemorrhage; MMC: Monash Medical Centre; NEC: Necrotizing Enterocolitis; NICU: Neonatal Intensive Care Unit; PCR: Polymerase Chain Reaction; PETS: Paediatric Emergency Transport Services; PEG: Percutaneous Endoscopic Gastrostomy; PICU: Paediatric Intensive Care Unit; PDA: Patent Ductus Arteriosus; RSV: Respiratory Syncytial Virus; PHT: Pulmonary Hypertension; ROP: Retinopathy of Prematurity; V-P Shunt: Ventriculo-Peritoneal Shunt
\end{abstract}

\section{Introduction}

Re-hospitalization after initial discharge from NICU for preterm infants has been examined in various settings across the world [1-13]. Reasons for re-hospitalizations in extreme preterm infants can be related either to medical (bronchiolitis, lower-respiratory tract infection, asthma, reflux disease) or to surgical (PEG related problems, V-P shunt related issues, hernia repair) causes. Researchers have attempted to ascertain neonatal 
$[1,3,9,12]$ and socioeconomic risk factors $[9,12,13]$ to establish at risk groups for readmission postneonatal discharge for very preterm and extremely preterm infants.

Within Australasia there are few studies highlighting subsequent outcomes of infants born preterm [1,2,9,1418]. Some of these papers were from the pre-surfactant era where neonatal care was significantly different from current neonatal care [15-18]. Others included very preterm infants (born before 32 weeks of gestation) and late preterm infants $[2,9]$. Some of them were based on weight rather than gestation age [1]. Hospital readmissions as an outcome of ELGAN is only reported in one study so far within Australasia [14].

We are presenting data from a geographically defined population in metropolitan Melbourne highlighting various aspects of re-hospitalizations including PICU admissions. Our main aim was to look for possible neonatal risk factors leading to postdischarge readmission. We aim to obtain data that will help us to counsel parents at the time of discharge from NICU.

\section{Methodology}

\section{Setting}

This study was carried out in Monash Children's Hospital, Clayton (MMC), which is a part of the Monash Health network. It is a tertiary care pediatric and perinatal referral centre in the south-east of Melbourne. Monash Health has a catchment area of six local government councils caring for a population of over one million Victorians. Monash Newborn is a tertiary perinatal and surgical unit where over 1000 newborns are admitted each year. Monash Children's provides tertiary pediatric care through Monash Medical Centre, Clayton (MMC) and secondary pediatric care through Dandenong General Hospital (DGH) and Casey General Hospital (CGH) and has over 30,000 yearly pediatric in patient episodes. MMC offers a full range of general pediatric, pediatric subspecialty and pediatric surgical inpatient and outpatient services including pediatric intensive care services. DGH and CGH offer general pediatric inpatient services mainly, while pediatricians review outpatients in their private rooms.

\section{Study population}

We included all newborns born on or before $27+6$ weeks of gestational age (Extremely low gestational age neonates) at MMC or transferred to our neonatal unit from the catchment area of Monash Health. Patients were identified using our neonatal database, which contributes prospective data to the Australia and New Zealand Neonatal Network (ANZNN) registry [19]. Patients were eligible for inclusion if they survived to discharge home and resided within our geographic catchment area. The most recent available address from either emergency department visits or outpatient visits or from inpatient admission records was used to judge eligibility of a neonate for this study.

\section{Study period}

Neonates born during the period of July 2010 to March 2013.

\section{Study design}

Retrospective cohort.

\section{Exclusion criteria}

1) Neonates born out of defined geographic location and 2) Neonates with major congenital malformations, as defined by AlHW [20].

\section{Data collection}

Demographic and neonatal course of the study population: After obtaining data from unit's ANZNN database we used our health network's Scanned Medical Record (SMR) to collect clinical and demographic data. Standard ANZNN data definitions were used. Birth details such as mode of delivery, gestational age, APGAR scores, birth weight, twin or higher order pregnancy were recorded from the neonatal discharge summary. Information on neonatal course such as duration of invasive mechanical ventilation required in $\mathrm{NICU}$, diagnosis of Bronchopulmonary Dysplasia (BPD), presence or absence of Patent Ductus Arteriosus (PDA) or Pulmonary Hypertension (PHT), and Intraventricular Hemorrhage (IVH) were recorded from observation charts, echocardiography reports and ultrasound reports. Discharge details such as duration of stay at MMC, mode of feeding, discharge weight and use of home oxygen were obtained from the discharge summary. Diagnosis of PDA and presence of pulmonary hypertension were based on the documented opinion of pediatric cardiologists. Presence of IVH and its grading were based on the cranial ultrasound assessment by the pediatric radiologist using the Papile classification [21]. Neonatal meningitis was diagnosed on the basis of abnormal Cerebrospinal Fluid (CSF) results and documented opinion from the pediatric infectious diseases specialists.

Inpatient admission: The health network's scanned medical record which is used across all three hospitals was used to review inpatient medical records. Data collected until corrected age of 18 months included: Number of admissions, admitting hospital, admitting team, admitting ward (Paediatric ICU, Paediatric HDU or Paediatric ward), any need for escalation of respiratory support, final diagnosis by the treating team, total days required for each admission, mortality if any and cause of death. Nasopharyngeal aspirates were sent in selected cases at the discretion of the treating physician. Viral identification is usually performed in our laboratory by PCR. We aimed to identify neonatal risk factors for re-hospitalization by comparing neonatal demographic for patients who were admitted to those who were not admitted. 
Corrected age at which oxygen was completely weaned off oxygen: Corrected age when home oxygen therapy was completely ceased was obtained from scanned medical records. Immunisation records and records regarding Palivizumab were also obtained.

\section{Data analysis}

For continuous data, if data was not distributed normally than outcome was presented as median and range quartile or else mean and standard deviation was used. For categorical data frequency and percentage were used. Comparisons of binary outcomes were made by using Fisher's exact test. Comparisons of continuous data among 2 groups were made by using Mann-Whitney $U$ test. $P$ value was considered significant if it is below 0.05. We did not conduct Bonferroni's test to address the problem of mass significance.

\section{Ethics approval}

This study was approved by the hospital ethics committee (Ref No-14404 Q).

\section{Results}

\section{Study population}

A total of 127 ex-ELGAN patients were discharged alive during the prescribed study period. One baby was excluded in view of his associated complex congenital heart disease requiring transfer to a cardiac surgical hospital; one baby was not discharged from NICU even at 18 months of corrected age; and one baby was transferred to another tertiary care hospital and hence further neonatal data were not available. 48 babies were discharged to geographic regions outside of our hospital's catchment area. Finally, 76 newborns who were discharged to the geographical catchment area were included for this study (See the flow chart). Their clinical profile is summarized in Table 1.

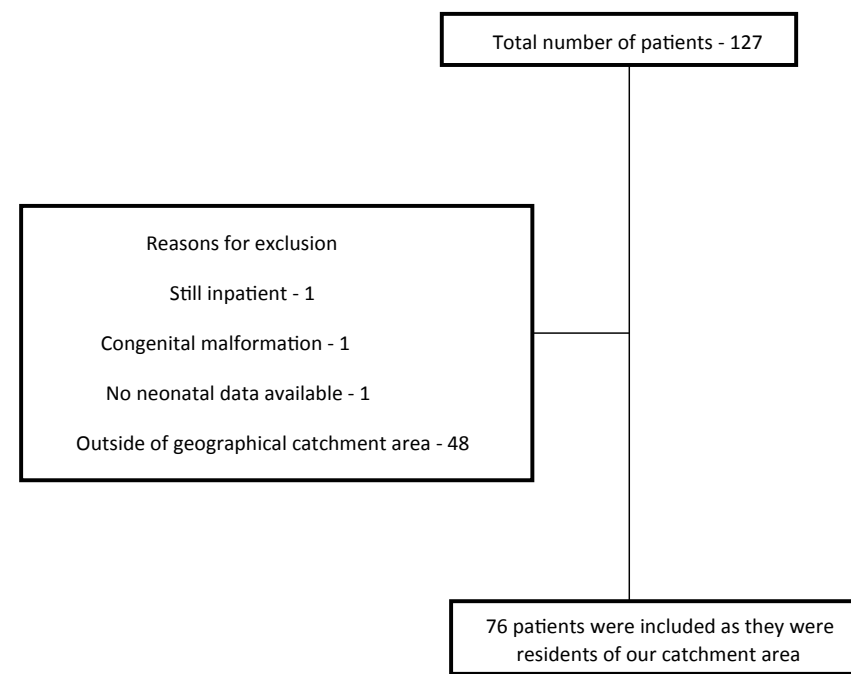

\section{Features of re-admission episodes}

Important features are highlighted in Table 2. 41 patients had 92 re-admission episodes which summed to a re-admission rate of $54 \%$. Out of these 19 (44\%) patients had just 1 admission, 8 patients had 2 admissions and 14 patients had 3 or more admissions within 18 months of their corrected age. The highest recorded number of admission episodes by a single patient was 6 .

\section{Admission diagnosis, admitting team and proce- dures performed during admissions}

Final diagnosis as recorded by treating team has been displayed in Figure 1. Respiratory causes were the leading discharge diagnosis $(71 / 92,68 \%)$, with bronchiolitis the most common diagnosis. Nasopharyngeal swab or aspirates were sent in 48 episodes of admissions related

Table 1: Neonatal and demographic data for study population.

Total number of patients in the study

M: F

Number of twin or part of twin (\%)

Number of doses of antenatal steroid

(number)

Median gestational age at birth (weeks)

Median birth weight (grams)

\begin{tabular}{|l|}
\hline 76 \\
\hline $44: 32: 00$ \\
\hline $15 / 76(19.73 \%)$ \\
\hline 2 doses $59 / 76$ \\
\hline 1 dose $9 / 76$ \\
\hline no dose $8 / 76$ \\
\hline 26 (range quartile 2$)$ \\
\hline $23-24$ weeks- 16 \\
\hline $25-26$ weeks- 40 \\
\hline 27 weeks- 20 \\
\hline 818.5 (range quartile 237$)$ \\
\hline Less than 500 grams -2 \\
\hline$\geq 500$ to $<750$ grams -26 \\
\hline$\geq 750-<1000$ grams -34 \\
\hline$\geq 1000$ grams - 14 \\
\hline 25.5 (range quartile 41$)$ \\
\hline $5 / 76(6.5 \%)$ \\
\hline $46 / 76$ (60.5\%) \\
\hline $5 / 76(6.5 \%)$ \\
\hline 102.5 , (range quartile 65.5$)$ \\
\hline 40 (IQR 7$)$ \\
\hline $36 / 76$ (47.3\%) \\
\hline
\end{tabular}

Median duration of invasive ventilation (days)

Number of patients requiring PDA ligation

Diagnosed Bronchopulmonary dysplasia (number)

Number of babies with major intraventricular bleed

Median Length of stays in MMC (days)

Median gestation at time of discharge from NICU (weeks)

Number of patients discharge with oxygen 
Table 2: In-patient admissions for study population.

Total number of admissions

Number of patients with 0 admission

Number of patients with 1 admission

Patients requiring 2 and more admissions

Median corrected age (months) of admission

Type of admission
92 (Range: 0-6; Median 1 and range quartile-2) $35 / 76(-46.05 \%)$

$19 / 76(-25 \%)$

$22 / 76(28.94 \%)$

5 (range quartile 8.7 )

Elective admission -22 (23.9\%)

Emergency department $-70(76.1 \%)$

Number of episodes where Paediatric Emergency Transport Services (PETS) 8 (8.6\%)

were used by

Number of admission to General Ward

$81 / 92(88 \%)$

Number of admission to ICU/HDU

$11 / 92(11.9 \%)$

Escalation of respiratory support Invasive ventilation -3 , required

Mortality during admission

CPAP -2, High flow -4 0

\section{Final diagnosis}

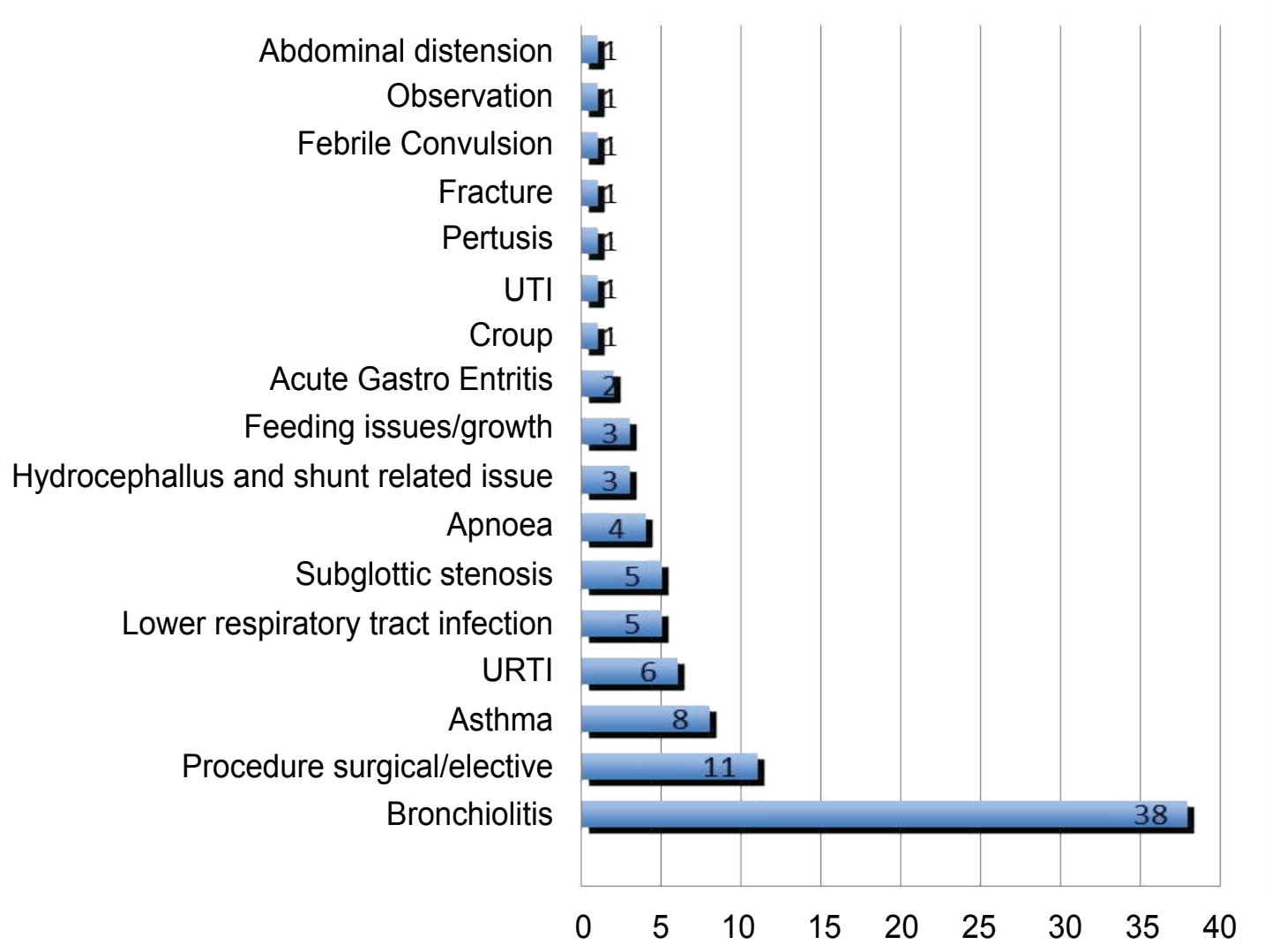

Figure 1: Final diagnosis during admission.

to respiratory causes, hence in 23 admission episodes swabs were not considered. Picornavirus was the most frequent virus isolated $(20 / 48,42 \%)$ followed by Respiratory Syncytial Virus (RSV) $(12 / 48,25 \%)$. Parainfluenza (3/48), Adenovirus (3/48), Influenza (1/48) and Bordetella Pertussis (1/48) were the other pathogens isolated. Samples were negative in 8 out of 48 samples. 20 elective procedures were recorded during 22 elective admission episodes as illustrated in Figure 2.

Most admissions occurred at MMC (82\%) while CGH (10\%) and DGH (8\%) provided a minor contribution. Nearly half (47 out of 92) of the patients were admitted under the general paediatric unit, followed by paediatric respiratory unit (25/92). Paediatric surgery (3/92), pediatric gastroen- terology (3/92), paediatric urology (2/92), ENT (4/92) and neonatology (4/92) were the other admitting units.

\section{Admissions with bronchiolitis}

A significant proportion of our admissions were related to bronchiolitis (38/92, 41\%). 25 patients had 38 admission episodes related to bronchiolitis, with 11 requiring two or more admissions. Median corrected age for admission related to bronchiolitis was 4.7 months (Range quartile 5.5). Median duration of admission for all the patients with bronchiolitis was 4 days (range 1 to 79 days, range quartile 6). Seven of these 38 required ICU admissions. RSV was isolated in 10, while Picornavirus was isolated in 13. In 14 out of $38(37 \%)$ episodes, patients were still receiving home 


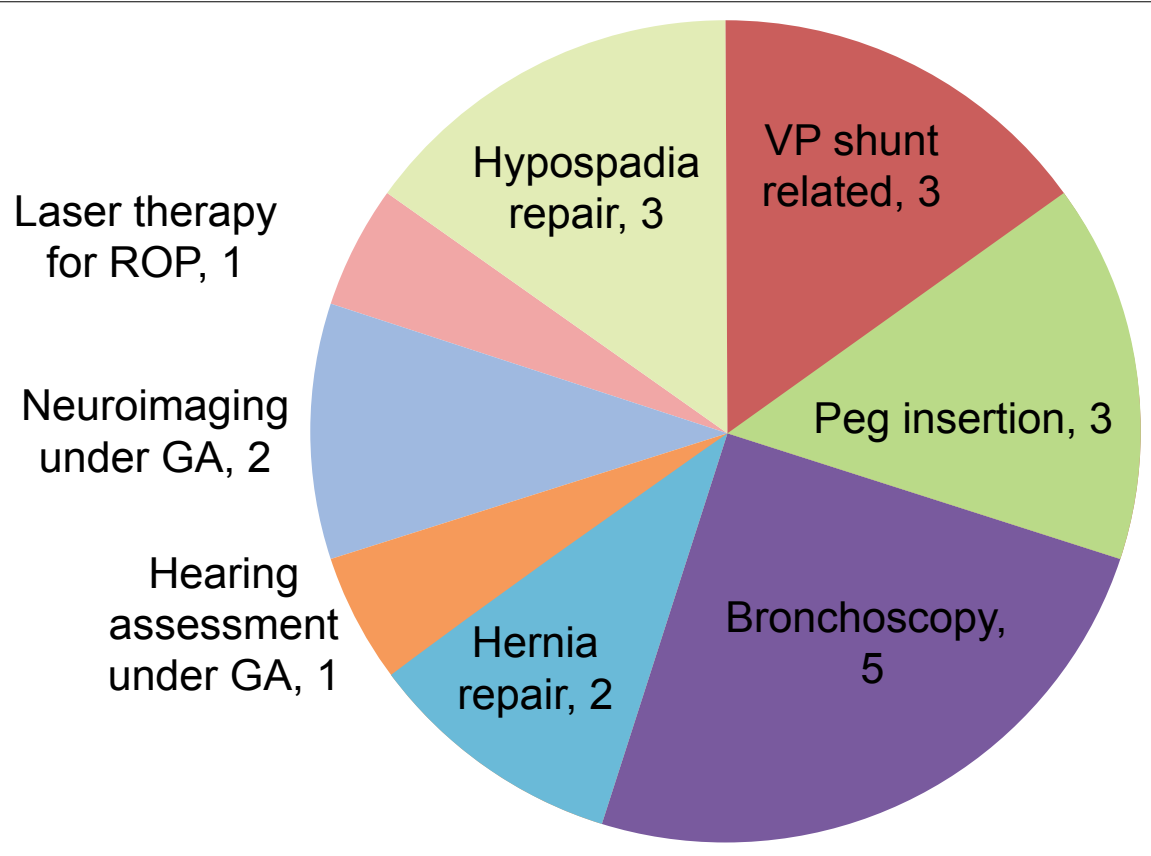

Figure 2: Various procedure performed during admissions.

Table 3: Features of patients requiring PICU admission.

\begin{tabular}{|l|l|}
\hline $\begin{array}{l}\text { Number of patients requiring PICU } \\
\text { admissions }\end{array}$ & 9 \\
\hline $\begin{array}{l}\text { Mean gestation of the admitted patients } \\
\text { Mean birth weight in grams }\end{array}$ & 26.1 (SD 1) \\
\hline $\begin{array}{l}\text { Number of male infants requiring PICU } \\
\text { admissions }\end{array}$ & $7 / 9(77.7 \%)$ \\
\hline $\begin{array}{l}\text { Twin pair } \\
\text { Number of patients diagnosed with } \\
\text { neonatal meningitis }\end{array}$ & $0 / 9$ \\
\hline $\begin{array}{l}\text { Number of patients diagnosed with BPD } \\
\text { at discharge from NICU }\end{array}$ & $5 / 9$ \\
\hline \begin{tabular}{l} 
Diagnosis of admitted patients in PICU \\
Bronchiolitis-7, \\
Subglottic \\
stenosis-2, \\
Pertussis-1 \\
\hline Median age (months) for admission (IQR)
\end{tabular} & $3.25(4.87)$ \\
\hline $\begin{array}{l}\text { Median duration of stay (days) in PICU } \\
\text { (IQR) }\end{array}$ & $7(1.5)$ \\
\hline $\begin{array}{l}\text { Number of patients discharged on home } \\
\text { oxygen from NICU }\end{array}$ & $6 / 9$ \\
\hline
\end{tabular}

oxygen therapy prior to their hospital admission related to bronchiolitis. Information about the immune prophylaxis for RSV was not consistently documented.

\section{Features of ICU admissions}

Features of ICU admissions have been described in Table 3. Nine patients required 10 ICU admissions (11\% of total admissions). Of the seven bronchiolitis admissions, 3 patients required invasive mechanical ventilation, 2 patients required CPAP and other 2 were managed with high flow.

Two out of 10 admissions were for subglottic stenosis and both of them required high flow as respiratory support. One admission was diagnosed to have severe pertussis and was managed with nasal high flow support.

\section{Risk factor for admission}

Possible risk factors and comparison of the demographic features have been displayed in Table 4. Pa-

Table 4: Possible risk factors for admission.

\begin{tabular}{|c|c|c|c|}
\hline & No admission & Admitted & p Value \\
\hline & $N=35$ & $N=41$ & \\
\hline \multirow[t]{2}{*}{ Gestational age (weeks) } & 25.8 & 25.46 & $0.25^{*}$ \\
\hline & (SD 1.0) & (SD 1.2) & \\
\hline \multirow[t]{2}{*}{ Average weight (grams) } & 820.28 & 837 & $0.88^{*}$ \\
\hline & (SD 151.1) & (SD 208.2) & \\
\hline Male child (number) & $19 / 35$ & $25 / 41$ & $0.64^{\#}$ \\
\hline Twin pairs (number) & $13 / 35$ & Feb-41 & $0.005^{\#}$ \\
\hline Meningitis in neonatal period & 0 & 7 & $0.02^{\#}$ \\
\hline \multirow[t]{2}{*}{ Duration of Mechanical ventilation (days) } & 20.85 & 31.7 & $0.09^{*}$ \\
\hline & (SD 20.53) & (SD 25.82) & \\
\hline BPD (number) & $19 / 35$ & $27 / 41$ & $0.35^{\#}$ \\
\hline \multirow[t]{2}{*}{ Average stay in NICU (days) } & 103.74 & 125.48 & $0.22^{*}$ \\
\hline & (SD 43.5) & (SD 67.8) & \\
\hline
\end{tabular}

\#Fisher's exact test; "Mann-Whitney U test. 
tients treated for meningitis in the neonatal period and singleton in comparison to twins was more likely to require hospital readmissions.

\section{Corrected age when oxygen was completely ceased post NICU discharge}

We have got data of 34 patients out of 36 patients who were discharged with home oxygen therapy from NICU. Median corrected age when oxygen was completely ceased was 4.5 months (range quartile - 5.5). Out of 34 patients 2 patients were still on home oxygen therapy even at 18 months of corrected age. One infant required high flow oxygen support at home.

\section{Discussion}

\section{Features of in-patients}

Our re-admission rate of $54 \%$ is quite similar to previously reported rates from various international settings 50\% $[1,3,14]$. Previous Australasian data describes a readmission rate of $42 \%$ for VLBW infants [9] and $55 \%$ for very preterm infants [2].

\section{Admission diagnosis}

Respiratory aetiologies as the predominant cause for hospital re-admission has been reported by many researchers $[1,2,13]$. Interestingly, bronchiolitis related to Picornavirus was more common in our study than RSV - a different pattern to previous reports [21,22]. RSV related bronchiolitis is an established outcome in infants born preterm. Unfortunately, we did not have reliable data on RSV immune prophylaxis, so we are unable to draw firm conclusions regarding its effect in our NICU graduate population.

In our cohort, procedure-related elective admissions were common as reported in other publications [13].

\section{ICU admissions}

Data about ICU admission in ex-ELGANs are not well reported in the literature. Mourani, et al. concluded that small preterm infants have substantial risk of re-admission in ICU [23]. In their study $30 \%$ of total admissions resulted in PICU and two thirds of these required mechanical ventilation for an average 11.6 days. Our patient cohort had a rate of ICU admission of $11 \%$ of total admissions and among these $30 \%$ of episodes required mechanical ventilation. The predictor of ICU admission in Mourani, et al. study was male sex and intraventricular hemorrhage. We had a higher number of males in patients admitted to the ICU (77.7\%), however, none of our patients requiring ICU admission had persistent abnormalities of their head ultrasounds. Four out of 10 (40\%) of ICU admissions in our study were related to RSV infection. Greenough, et al. in their retrospective review concluded that RSV hospitalization in patients with chronic lung disease is associated with increased ICU admission, and longer duration of ICU stay [6].

\section{Risk factors for hospital re-admission}

Various studies across the world have tried to ascertain the neonatal risk factor for post NICU discharge hospital re-admission [1-3,8-13]. Ambalavanan, et al. in their recent publication using classification and regression tree analysis concluded that neonates who spent $>120$ days during their NICU admission for pulmonary reasons had a $66 \%$ re-hospitalization rate compared to $42 \%$ without such stay [3]. Among studies from Australasia, Elder, et al. found diagnosis of chronic lung disease during NICU admission as a significant risk factor for hospital re-admission [9], while Gray, et al. in their prospective analysis, though concluded that preterm infants required higher rate of hospital readmission, they were unable to find any specific neonatal risk factor responsible for higher re-admission rate. In our cohort we found meningitis in neonatal period was more common in patients who required hospital re-admission. This finding can be explained by the fact that meningitis in neonatal period will certainly prolong the NICU stay which is an established risk factor for subsequent post NICU discharge re-admission. However, as we had a smaller number of patients in our cohort, we would like to interpret this finding with caution. We also find that twins were less likely to require re-admission in comparison to singleton, we believe this finding is a result of smaller number of twin included in the study.

\section{Corrected age when home oxygen was completely weaned off}

Home oxygen therapy in preterm infants is an interesting and perhaps under-reported outcome. Within Australasia, we could find 3 studies highlighting this outcome [24-26]. Complete weaning off home oxygen therapy varied between 4.5 to 6 months of corrected age in these studies. This is quite comparable with our study.

\section{Strengths and Limitation of the Study}

Study was conducted at a tertiary referral centre with availability of almost all pediatric specialties making data more reliable. Though our sample size was also small our findings are consistent with findings reported from elsewhere. Viral isolation for respiratory diagnosis is strength of this study. The limitations of our study were its retrospective design of chart review. Although this can be subject to bias [27]. We attempted to minimize this by structured, reproducible methodology and restricting data collection to reliably recorded outcomes. Many patients were discharged out of our health service's geographical catchment area; these excluded patients may reduce the external validity of our results. Unfortunately, socio-economic data and RSV immune prophylaxis were not consistently recorded for many patients. We did not consider the effect of mass significance in our analysis this perhaps could affect the overall result. 


\section{Conclusion}

Hospital re-admission for infants born at extremely low gestational age is common. Respiratory conditions are the most common reason for re-admission to the ward and PICU. The majority of PICU admissions are related to respiratory conditions, particularly bronchiolitis. Neonatal meningitis was more common in patients who required re-admission following NICU discharge. We propose a case registry or a prospective study to address ongoing uncertainty relating to predictors of outcomes for ELGANs.

\section{References}

1. Doyle LW, Ford G, Davis N (2003) Health and hospitalisations after discharge in extremely low birth weight infants. Semin Neonatol 8: 137-145.

2. Gray D, Woodward LJ, Spencer C, Inder TE, Austin NC (2006) Health service utilisation of a regional cohort of very preterm infants over the first 2 years of life. J Paediatr Child Health 42: 377-383.

3. Ambalavanan N, Carlo WA, McDonald SA, Yao Q, Das A, et al. (2011) Identification of extremely premature infants at high risk of rehospitalization. Pediatrics 128: e1216-e1225.

4. Greenough A, Alexander J, Burgess S, Bytham J, Chetcuti PA, et al. (2006) Preschool healthcare utilisation related to home oxygen status. Arch Dis Child Fetal Neonatal Ed 91: 337-341.

5. Greenough A, Alexander J, Boorman J, Chetcuti PA, Cliff I, et al. (2011) Respiratory morbidity, healthcare utilisation and cost of care at school age related to home oxygen status. Eur J Pediatr 170: 969-975.

6. Greenough A, Cox S, Alexander J, Lenney W, Turnbull F, et al. (2001) Health care utilisation of infants with chronic lung disease, related to hospitalisation for RSV infection. Arch Dis Child 85: 463-468.

7. Greenough A, Limb E, Marston L, Marlow N, Calvert S, et al. (2005) Risk factors for respiratory morbidity in infancy after very premature birth. Arch Dis Child Fetal Neonatal Ed 90: 320-323.

8. Underwood MA, Danielsen B, Gilbert WM (2007) Cost causes and rates of rehospitalization of preterm infants. $J$ Perinatol 27: 614-619.

9. Elder DE, Hagan R, Evans SF, Benninger HR, French NP (1999) Hospital admissions in the first year of life in very preterm infants. J Paediatr Child Health 35: 145-150.

10. Stephens BE, Tucker R, Vohr BR (2010) Special health care needs of infants born at the limits of viability. Pediatrics 125: $1152-1158$.

11. Korvenranta E, Lehtonen L, Peltola M, Häkkinen U, Andersson S, et al. (2009) Morbidities and hospital resource use during the first 3 years of life among very preterm infants. Pediatrics 124: 128-134.

12. Lamarche-Vadel A, Blondel B, Truffer $P$, Burguet A, Cambonie G, et al. (2004) Re-hospitalization in infants younger than 29 weeks'gestation in the EPIPAGE cohort. Acta Paediatr 93: 1340-1345.

13. Morris BH, Gard CC, Kennedy K, NICHD Neonatal Research Network (2005) Rehospitalization of extremely low birth weight (ELBW) infants: are there racial/ethnic disparities? J Perinatol 25: 656-663.
14. Stephens AS, Lain SJ, Roberts CL, Bowen JR, Nassar N (2016) Survival, Hospitalization, and Acute-Care Costs of Very and Moderate Preterm Infants in the First 6 Years of Life: A Population-Based Study. J Pediatr 169: 61-68.e3.

15. Ford GW, Rickards AL, Kitchen WH, Lissenden JV, Keith CG, et al. (1985) Handicaps and health problems in 2 year old children of birth weight 500 to $1500 \mathrm{~g}$. Aust Paediatr J 21: 15-22.

16. O'Callaghan MJ, Burns Y, Gray P, Harvey JM, Mohay HI, et al. (1995) Extremely low birth weight and control infants at 2 years corrected age: A comparison of intellectual abilities, motor performance, growth and health. Early Hum Dev 40: $115-128$

17. Bowman E, Yu VY (1988) Continuing morbidity in extremely low birthweight infants. Early Hum Dev 18: 165-174.

18. Cunningham CK, McMillan JA, Gross SJ (1991) Rehospitalization for respiratory illness in infants of less than 32 weeks' gestation. Pediatrics 88: 527-532.

19. Chow SSW, Le Marsney R, Hossain S, Haslam R, Lui K (2015) Report of the Australian and New Zealand Neonatal Network 2013. ANZNN, Sydney, 1-100.

20. Abeywardana S, Sullivan EA (2008) Congenital anomalies in Australia 2002-2003. Birth anomalies series no. 3 Cat. no. PER 41. AIHW National Perinatal Statistics Unit, Sydney, 1-165.

21. Papile LA, Burstein J, Burstein R, Koffler H (1978) Incidence and evolution of subependymal and intraventricular hemorrhage: a study of infants with birth weights less than 1,500 gm. J Pediatr 92: 529-534.

22. Li QH, Gao WJ, Li JY, Shi LA, Hao XJ, et al. (2016) [Detection of respiratory viruses in children with acute lower respiratory tract infection: an analysis of 5,150 children]. Zhongguo Dang Dai Er Ke Za Zhi 18: 51-54.

23. Calvo C, Pozo F, García-García ML, Sanchez M, Lopez-Valero M, et al. (2010) Detection of new respiratory viruses in hospitalized infants with bronchiolitis: A three-year prospective study. Acta Paediatr 99: 883-887.

24. Mourani PM, Kinsella JP, Clermont G, Kong L, Perkins AM, et al. (2014) Intensive care unit readmission during childhood after preterm birth with respiratory failure. J Pediatr 164: 749-755.e3.

25. Saletti A, Stick S, Doherty D, Simmer K (2004) Home oxygen therapy after preterm birth in Western Australia. J Paediatr Child Health 40: 519-523.

26. Silva DT, Hagan R, Sly PD (1995) Home oxygen management of neonatal chronic lung disease in Western Australia. J Paediatr Child Health 31: 185-188.

27. Gilbert EH, Lowenstein SR, Koziol-McLain J, Barta DC, Steiner J (1996) Chart reviews in emergency medicine research: Where are the methods? Ann Emerg Med 27: 305-308. 